\title{
EDITORIAL
}

\section{Participatory medicine: a driving force for revolutionizing healthcare}

\author{
Leroy Hood ${ }^{1 *}$ and Charles Auffray ${ }^{2^{*}}$
}

Healthcare is undergoing a profound revolution as a consequence of three contemporary thrusts: systems medicine [1-4], big data and patient involvement in their own health through social networks. This convergence is leading to a medicine that is predictive, preventive, personalized and participatory (P4) [4-7]. The first three Ps, predictive, preventive and personalized, were delineated in the early 2000 s $[1,2]$, whereas the fourth $\mathrm{P}$, participatory, was added later. To achieve a participatory healthcare system, major technical and societal challenges will need to be overcome, and this will require close integration with systems medicine and big data. Before commenting further on participatory medicine, let us first delineate the essence of systems medicine and big data and their implications for P4 medicine.

Systems medicine, the application of systems biology approaches to disease $[3,4,6,8,9]$, is already changing how healthcare is practiced (Table 1). We predict that in 5 to 10 years each patient will be surrounded by a virtual cloud of billions of data points - molecular, clinical chemistries, cellular, organ, phenotypic, imaging, social networks, and so on. We also predict that we will have the analytical tools to reduce this big data cloud to simple models that will guide improvements in health and minimize disease. This requires addressing two grand challenges: the signal-to-noise issues intrinsic to big data and integration of multi-scale data into predictive models.

In addition, for each individual patient we foresee that we will be able to integrate an individual's genetic, molecular, cellular, organ and social networks into an overall 'network of networks' [10]. Disease leads to the perturbation of the network of networks and this alters the information the network manages. The availability of a personal data cloud will allow one to characterize an

\footnotetext{
*Correspondence: Ihood@systemsbiology.org; cauffray@eisbm.org 'Institute for Systems Biology, P4 Medicine Institute, 401 Terry Avenue North, Seattle, WA 98109, USA

EEuropean Institute for Systems Biology \& Medicine, CNRS-ENS-UCBL, Université de Lyon, 50, Avenue Tony Garnier, 69007, Lyon, France Full list of author information is available at the end of the article
}

individual's network of networks in its normal or disease-perturbed states. This altered, disease-perturbed information will provide deep insights into disease mechanisms, new approaches to diagnostics and therapeutics, and a platform for participatory medicine.

\section{Participatory medicine}

Implementation of $\mathrm{P} 4$ medicine relies on achieving two major objectives. First, the technical aspects of systems medicine - strategies, technologies, analytical tools -must be developed so that big data can be generated and analyzed to reveal the dynamic networks for each patient. The technical solutions to these challenges are advancing very effectively. Second, the societal challenges of $\mathrm{P} 4$ medicine must be solved - and in our mind these represent far greater hurdles for the acceptance of P4 medicine into healthcare systems [11]. These societal challenges come in eight forms, as follows.

First, there are the obvious questions that relate to the ethical, legal, societal, security, privacy, policy, regulatory aspects and economics of $\mathrm{P} 4$ medicine that deal with individual patients. These have been discussed extensively elsewhere [12].

Second, there is the fascinating and difficult question of who is included in the term 'participatory'. Obviously patients must be included because a core idea of personalized medicine is that patients increasingly take control of their own health. However, to integrate P4 medicine into healthcare systems, we must also include physicians and other healthcare workers, other members of the healthcare system (payers, providers), those concerned with policy and implementation of healthcare, and those involved with education of students and the general public. Bringing all these participants into alignment around P4 medicine is an enormous challenge.

Third, ultimately bringing these participants into alignment will necessitate a change in education and consumer feedback - how do we bring an appropriate understanding of $\mathrm{P} 4$ medicine to all participants in the healthcare system? And how do we systematically obtain 
Table 1 How systems medicine is transforming healthcare

Systems medicine
Provides fundamental insights into dynamic disease-perturbed networ
in model organisms
Pioneers family genome sequencing, identifying disease genes
inexpensively and effectively
Transforms blood into a window to distinguish health from disease

Stratifies diseases into their distinct subtypes

Stratifies patients - drug adverse reactions, modifier genes to disease mechanisms, for example early and late onset of Huntington's disease, variant genes that increase mercury susceptibility in children

Permits a multi-organ approach to the study of disease

Enables new computational approaches to pioneering drug reuse and drug target discovery

Focuses on wellness

Creates large-scale, multiparameter, Framingham-like clinical trials to permit the longitudinal analyses of disease or wellness states

\section{Healthcare outcome}

Enables mechanistic insights, diagnosis, therapy and prevention for the individual patient

Identifies disease, wellness and drug-intolerant gene products. Identifies individuals who have any one or more of 300 actionable gene variants

Disease diagnostics, assesses drug toxicity and wellness; examples include lung cancer, post-traumatic stress disorder (PTSD), liver toxicity and hepatitis

For selecting specific and effective drugs for each subtype

Stratifies patients for appropriate treatments

Unravels the complexity of the individual patient's disease and how a single disease affects multiple organs

Re-engineers disease-perturbed networks to normalcy with drugs and repurpose drugs. Faster and cheaper to develop drugs that prevent networks from becoming disease-perturbed

Wellness is a driver for P4 medicine of the future

These studies will provide insights into early disease mechanisms and will provide new approaches to diagnosis and therapy their feedback for guidance in developing new wellness products and services? Our feeling is that these enormous challenges can only be solved by employing powerful IT tools, such as the massive online open courses that are transforming the practice of higher education, bringing it within reach of much larger numbers of individuals [13]. Indeed, we submit that the IT for healthcare of the future should include the means for educating all participants.

Fourth, it is essential to educate patients regarding the value of their data and how it can advance biomedical science without injury to them. A key to the continuous improvement of $\mathrm{P} 4$ medicine is the ongoing mining of the personal data clouds of billions of individuals. For this will allow rapid progress in our understanding of the complexities of disease and wellness - and profoundly change healthcare. Hence after appropriate deidentification, it is essential that each patient's data cloud be available for mining for the predictive medicine of the future. The narrow and restrictive institutional ethical review board policies of today must be replaced with open access to data for qualified professionals - with appropriate protection for the individual.

Fifth, patient (consumer)-activated social networks will play several important roles in assuring that patients will be empowered to participate actively in P4 medicine. Several such networks are already changing medicine, and one example is the 'quantified self' networks that have now spread widely in the United States. Individuals in these networks use digital devices to measure their own physical parameters (weight, pulse, respiration, quality of sleep, stress, and so on) and use them to optimize health $[14,15]$. There are also many diseaseoriented social networks (for example, PatientsLikeMe, Breast Cancer Alliance, Multiple Myeloma Research Foundation) that are already beginning to alter how clinical trials are carried out [5]. These social networks illustrate that healthcare and wellness can move away from hospitals and into foundations and even the home. This decentralization of medicine is an important aspect of the emerging P4 medicine focus on wellness.

Sixth, our view is that patient-activated social networks will be one of the most powerful forces in driving the acceptance of P4 medicine. Already patients are beginning to question whether they want to have physicians who do not know anything about their genomes, or nutrition or wellness. Patients are beginning to move to more recently trained physicians knowledgeable of the new technologies and strategies that are transforming the practice of medicine. A challenging question is how to generate the patient-activated social networks that will trigger the healthcare systems to accept P4 medicine.

Seventh, it is clear that the participatory aspect of P4 medicine is extremely dependent upon first class IT for healthcare capable of managing and integrating millions to billions of personal data clouds - that is aggregating, mining, integrating them and finally producing models for each patient that are predictive and actionable - thus defining the future architecture of P4 medicine. In addition, it is essential that there be a 'trusted third party site' that contains up-to-date and accurate information on systems medicine and P4 medicine. Too often patients come to their physicians with incorrect 
information that has been obtained from the Internet. There needs to be an accurate and trusted source of information - for patients, physicians and healthcare workers.

Eighth, it is clear that if the information from patient data clouds is to be used effectively to optimize their wellness and minimize disease, there needs to be a trusted interpreter of these data clouds for each patient. It is our view that a new healthcare professional, which we term a healthcare and wellness coach, will need to be created who can look at the patient's data cloud and present it to them in a way that encourages them to use their data to improve their health. We believe that nursing and general medical practice would be an ideal starting background from which to train such individuals.

\section{The way forward}

To take P4 medicine forward, we can learn from our experience of implementing systems medicine [16]. We believe that there are several paths forward.

First, we can create P4 pilot programs that recruit large numbers of patients to use the principles of $\mathrm{P} 4$ medicine. At the Institute for Systems Biology (ISB) and the European Institute for Systems Biology and Medicine (EISBM), we are in the process of initiating such pilot programs focused on wellness - with many measurements made over an extended time period of 10 to 20 years. These pilot programs will demonstrate the power of P4 medicine to study wellness and wellness-todisease transitions while at the same time empowering patients and engaging all healthcare system stakeholders. For these programs, we need to develop an appropriate IT framework, learn to train healthcare and wellness coaches, create effective and integrated data generation platforms and pioneer the analytical tools that will allow the creation of predictive and actionable models for each participant's data cloud. Wellness pilot projects are thus ideal to join patients, physicians, consumers and other people within the healthcare system.

Second, we suggest creating an Institute for Systems Medicine at medical schools or academic healthcare institutions as a powerful way to bring the principles of P4 medicine to medical research, training and practice. Such an endeavor is an expensive but a transformational opportunity. ISB has pioneered the creation of such an institute at the University of Luxembourg (the Luxembourg Center for Systems Biomedicine) with great success.

Finally, one of the most powerful ways to push forward the P4 agenda is to create effective patient (consumer)-activated networks, initially in the context of wellness pilot programs.

\section{Coda}

The coming revolution in medicine is with us already $[6,12]$. Several terms have been coined for this paradigm change in healthcare - personalized medicine, precision medicine and P4 medicine (systems medicine). Personalized medicine alone does not delineate important additional features of this revolution - the predictive, preventive and participatory aspects. Precision medicine is a poor term as big data - a central feature of P4 medicine - is not (yet) precise. There are enormous signal-tonoise ratio challenges. Moreover, precision medicine does not properly describe any of the four central features of the new medicine. We thus prefer the term P4 medicine because it captures the essence of this paradigm change - especially the participatory aspect that we have discussed here and see as central to moving forward with the revolution in healthcare and wellness. Let us emphasize again in concluding that we see patient (consumer)-activated social networks as one of the principle driving forces for the acceptance of $\mathrm{P} 4$ medicine into the healthcare system. The challenge is how to generate effective patient-activated social networks centered on the requirements and opportunities of $\mathrm{P} 4$ medicine [17].

\section{Competing interests}

The authors declare that they have no competing interests.

\section{Published: 23 December 2013}

\section{References}

1. Hood L, Heath JR, Phelps ME, Lin B: Systems biology and new technologies enable predictive and preventative medicine. Science 2004, 306:640-643.

2. Weston AD, Hood L: Systems biology, proteomics, and the future of health care: toward predictive, preventative, and personalized medicine. J Proteome Res 2004, 3:179-196.

3. Auffray C, Chen Z, Hood L: Systems medicine: the future of medical genomics and healthcare. Genome Med 2009, 1:2.

4. Hood L, Flores MN: A personal view on systems medicine and the emergence of proactive P4 medicine: predictive, preventive, personalized and participatory. Nat Biotechnol 2012, 29:613-624.

5. Hood L, Friend SH: Predictive, personalized, preventive, participative (P4) cancer medicine. Nat Rev Clin Oncol 2011, 8:184-187.

6. Hood L, Balling R, Auffray C: Revolutionizing medicine in the 21st century through systems approaches. Biotechnol J 2012, 7:992-1001.

7. Sobradillo P, Pozo F, Agustí A: P4 medicine: the future around the corner. Arch Bronconeumol 2011, 47:35-40.

8. Nicholson JK: Global systems biology, personalized medicine and molecular epidemiology. Mol Syst Biol 2006, 2:52.

9. Loscalzo J, Barabasi AL: Systems biology and the future of medicine. Wiley Interdiscip Rev Syst Biol Med 2011, 3:619-627.

10. Goh Kl, Cusick ME, Valle D, Childs B, Vidal M, Barabasi AL: The human disease network. Proc Natl Acad Sci U S A 2007, 104:8685-8690.

11. Kitano $H$, Ghosh $S$, Matsuoka $Y$ : Social engineering for virtual 'big science' in systems biology. Nat Chem Biol 2011, 7:23-326.

12. Flores M, Glusman G, Brogaard K, Price ND, Hood L: P4 medicine: how systems medicine will transform the healthcare sector and society. Personalized Med 2013, 10:565-576.

13. Milgrom $\mathrm{H}$, Tran ZV: The rise of health information technology. Curr Opin Allergy Clin Immunol 2010, 10:178-180

14. Smarr L: Quantifying your body: a how-to guide from a systems biology perspective. Biotechnol J 2012, 7:980-991. 
15. Chen R, Mias Gl, Li-Pook-Than J, Lam HY, Chen R, Miriami E, Karczewski KJ, Hariharan M, Dewey FE, Chang Y, Clark MJ, Im H, Habegger L, Balasubramanian S, O'Huallachain M, Dudley JT, Hillenmeyer S, Haraksingh R, Sharon D, Euskirchen G, Lacroute P, Bettinger K, Boyle AP, Kasowski M, Grubert F, Sek S, Garcia M, Whirl-Carrillo M, Gallardo M, Blaso MA, et al: Personal omics profiling reveals dynamic molecular and medical phenotypes. Cell 2012 148:1293-1307.

16. Hood L: Acceptance remarks for Fritz J. and Delores H. Russ Prize. NAE J Bridge 2011, 41:46-49.

17. Lejbkowicz I, Caspi O, Miller A: Participatory medicine and patient empowerment towards personalized healthcare in multiple sclerosis. Expert Rev Neurother 2012, 12:343-352.

doi:10.1186/gm514

Cite this article as: Hood and Auffray: Participatory medicine: a driving force for revolutionizing healthcare. Genome Medicine 2013 5:110. 